\title{
Response priming effects in a digit naming task as a function of target-noise separation
}

\author{
JOHN H. FLOWERS \\ University of Nebraska, Lincoln, Nebraska 68588
}

\begin{abstract}
Oral naming latencies were measured for visually displayed digits that were flanked by either compatible (identical) or incompatible (different) noise digits. When target-noise spatial separation was "narrow" (1.1-deg visual angle), substantially longer latencies were observed for incompatible target-noise combinations than for compatible combinations, and this effect was greatest when the onset of the noise preceded the target by $150 \mathrm{msec}$. When target-noise spatial separation was increased to $3.3 \mathrm{deg}$, noise-compatibility effects were substantially attenuated when the noise preceded the target by $100 \mathrm{msec}$ or less, but the effects reappeared with longer SOA values and reached a maximum when the noise preceded the target by $250 \mathrm{msec}$. This three-way interaction of spatial separation, SOA, and noise compatibility offers support for the continuous flow conception of visual information processing described by Eriksen and Schultz (1979).
\end{abstract}

When human subjects are required to identify or classify a visual field containing "noise" elements similar to the target, the level of performance is generally less than that obtained with a "noiseless" field. Such interference effects, which occur even when the spatial location of the target is prespecified (Eriksen \& Eriksen, 1974), appear to reflect a combination of "early" or perceptual-level interactions (Bjork \& Murray, 1977; Estes, 1974) and interactions occurring at the response level following a detailed processing of both the target and the noise (Eriksen \& Schultz, 1979). The relative contribution of perceptual-level vs. responselevel effects of noise would appear to depend on the particular combination of stimulus display parameters and response structure used in a given task (Santee \& Egeth, 1980). The present study is concerned with the generation of response-level interactions and how their time course may provide information about the rate of accumulation of visual information as a function of retinal location.

\section{Continuous Flow}

Eriksen and Schultz (1979) described a model of information accumulation by the visual system that they have termed the continuous flow conception. According to this view, information is gathered continuously by the visual system over a period of time, and the rate of the information accumulation is dependent upon the quality of the information presented. Information falling on different retinal locations is accumulated in parallel, although the rate of that accumulation will be influenced by the clarity of the stimulus and the sensi-

This research was funded in part by NSF Grant IST-11162 to John H. Flowers and by a summer research fellowship grant from the University of Nebraska Research Council. tivity of the retinal location upon which it falls. Response priming occurs concomitantly with this parallel information accumulation, and the response to the target is therefore inhibited by the number of alternative competing responses and the degree of priming that they receive. According to continuous flow, successful attentional focusing (i.e., the ability to ignore surrounding distractors with no cost in response time) is in large measure the result of accelerating the activation processes associated with the target relative to those associated with the noise. Eriksen and Schultz (1979) provided experimental evidence supporting this view by showing that manipulations of the relative stimulus quality (e.g., size and contrast) of the noise and target influenced the magnitude of target-noise compatibility effects, and also by showing that noise elements exert their maximum effect when they lead the target element in time.

A strong prediction of the continuous flow conception that Eriksen and Schultz (1979) did not investigate is a triple interaction between target-noise spatial separation, the onset asynchrony between the noise and the target, and the response compatibility of the noise and target. Increasing the spatial separation between noise and target should reduce the differences in RT obtained on trials involving compatible and incompatible noise as long as the relative onset times of the target and noise remain constant. Such effects of target-noise separation have, in fact, been found in several previous experiments (e.g., Eriksen \& Hoffman, 1973). Such findings reflect, according to continuous flow, the fact that the noise elements are processed more slowly because they fall on an area of the visual field that is less sensitive. ${ }^{1}$ However, this reduction in the effects of target-noise compatibility should be offset by increasing the time between the initial appearance of the noise elements and the appearance of the target. A "head start" in processing 
the noise elements would presumably compensate for the slower processing time associated with the more distant retinal location, allowing the noise to reach a level of response priming sufficient to produce response competition with the target.

As opposed to a manual classification task in which the subject manually "sorts" targets into categories defined by the experimenter, the present experiment used a digit naming task for which voice RT was the dependent variable. This response modality was selected because it involves highly overlearned and automatic associations that are likely to be more sensitive to response priming effects than is manual classification (e.g., see Flowers, Warner, \& Polansky, 1979). Furthermore, the use of a highly overlearned response structure is less likely to be subject to change during the time course of the experiment, an important consideration when one intends to compare the shapes of functions obtained under different experimental conditions.

\section{METHOD}

\section{Subjects}

Six graduate students at the University of Nebraska-Lincoln (two females) were each paid $\$ 6$ for participating in two experimental sessions lasting approximately $90 \mathrm{~min}$ each. Subjects had normal or corrected vision, and English was their native language.

\section{Apparatus}

Stimuli were displayed on a Tektronix Model 604 display scope employing a P31 phosphor. The display was controlled by an Automated Data Systems $1800 \mathrm{~A}$ computer, which also timed subjects' responses and stored the latencies. A Lafayette voice relay served as the response device. The display scope, a microphone, the voice relay, and a telegraph key for response initiation were all located on a small table at which the subject was seated. The distance between the scope and the subjects' eyes was not fixed by a restraining device, but, given the position of the chair, the viewing distance averaged approximately $45 \mathrm{~cm}$, with little variation among subjects.

\section{Conditions}

The three independent variables investigated in Experiment 1 were the distractor type (congruent vs. incongruent, plus a nonoise control), the onset asynchrony (SOA) between the distractors and the target $(0,50,100,150,200,250$, or $300 \mathrm{msec})$, and spatial separation (wide, $3.3 \mathrm{deg}$ between target and distractors, vs. narrow, $1.1 \mathrm{deg}$ separation between target and distractors). In each of the two experimental sessions, subjects responded to 16 different blocks, each containing 45 trials. For half the subjects, the first eight blocks of trials included stimuli with the narrow target distractor separation followed by eight blocks containing stimuli with the side target distractor separation; this order was reversed during the second experimental session. The remaining subjects received the complementary ordering of target-distractor separations.

Each of the eight blocks of trials during which targetdistractor separation was held constant consisted of one block for each of the seven values of SOA, plus one control block in which no distractors occurred (i.e., the flanking elements remained zeros). Within this control block, the center target appeared $900 \mathrm{msec}$ after the keypress on each trial; thus the effective "SOA" for the control block was $100 \mathrm{msec}$. Distractor type (congruent vs. incongruent) was the only independent variable that varied within a block of 45 trials (except for the control list). The first three trials in each block were practice trials omitted from data analysis. The remaining 42 trials each contained 21 "congruent" displays (three each of $222,333, \ldots$, 888 ) and 21 incongruent examples (e.g., 323, 262, 878, etc.). The digits 2-8 each occurred three times among these 21 incongruent stimuli in each block. The actual ordering of stimuli within each block was determined by a pseudorandom process, and the order of SOAs, plus the position of the control block during each eight-block half-session, was determined by block randomization for each subject on each day.

\section{Procedure}

Prior to beginning an experimental session, each subject was seated at the table in the dimly lit experimental room for approximately a 5 -min period in order to allow dark adaptation. During a block of trials, subjects initiated each display sequence by pressing the telegraph key. The key depression triggered the display of a fixation field consisting of a horizontal row of three zeros centered in the screen. These three zeros marked the positions in which the stimulus display (two flanking distractors and the target) were to appear. Assuming a viewing distance of $45 \mathrm{~cm}$, each of these zeros subtended a vertical visual angle of approximately $.9 \mathrm{deg}$ and were horizontally separated by either $1.1 \mathrm{deg}$ or $3.3 \mathrm{deg}$ of visual angle between the centers of each zero, depending upon the experimental conditions, as described below. The flanking zeros each changed into a digit (2-8) $800 \mathrm{msec}$ after the appearance of the fixation row. These digits constituted the distractor elements. The center zero was then replaced by the target digit (2-8) after a delay of $0-300 \mathrm{msec}$. This delay constituted the SOA. ${ }^{2}$

Subjects responded by vocalizing the name of the digit in the center position "as rapidly as possible, avoiding errors." The triggering of the voice relay terminated the screen display and instructed the computer to store the response latency. The experimenter followed the subject's responses with an answer key and marked any trials in which an error was made, or on which any stuttering or premature vocalization occurred. Such errors, although infrequent (under $3.0 \%$ on the least accurate condition), were tabulated, but latencies from those trials were not included in data analysis.

\section{RESULTS}

\section{Reaction Times}

Mean RTs were computed for each subject for each combination of distractor type, SOA, and spatial separation, and also for the distractorless control stimuli for the two spatial separations. Mean RTs across subjects for each level of SOA and target-noise compatibility are shown in Figure 1 (for the narrow spatial separation) and in Figure 2 (for the wide spatial separation). As these figures suggest, there was an overall effect of noise type that was statistically significant $[F(1,5)=$ $13.2, \mathrm{p}<.05]$ and, additionally, a main effect of spatial separation $[F(1,5)=11.4, p<.05]$. SOA also produced a main effect $[F(6,30)=5.6, p<.01]$, which may reflect a general warning signal or alerting property of the distractors regardless of their compatibility with the target.

The results of major interest, however, are the twoway interaction between noise type and $\operatorname{SOA}[F(6,30)=$ $3.51, \mathrm{p}<.01]$ and the three-way interaction of spacing, noise type, and $\operatorname{SOA}[F(6,30)=6.14, p<.01]$. It is 


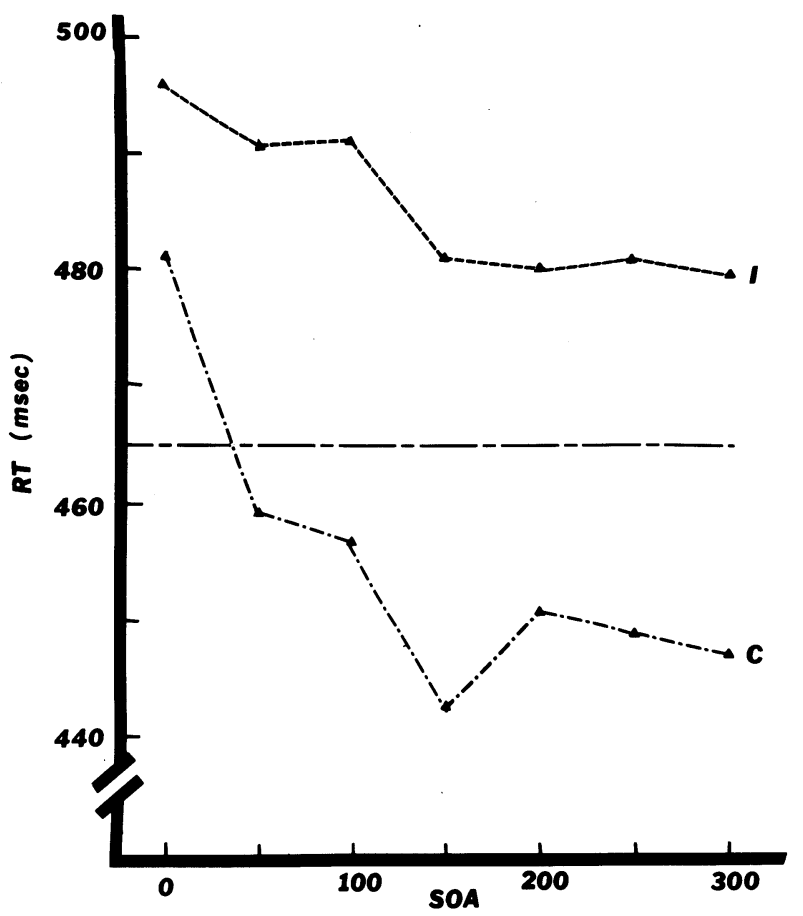

Figure 1. Mean RTs as a function of SOA for the "narrow" displays. $I=$ incongruent; $C=$ congruent. Baseline $R T$ for the distractorless stimuli is indicated by the horizontal line.

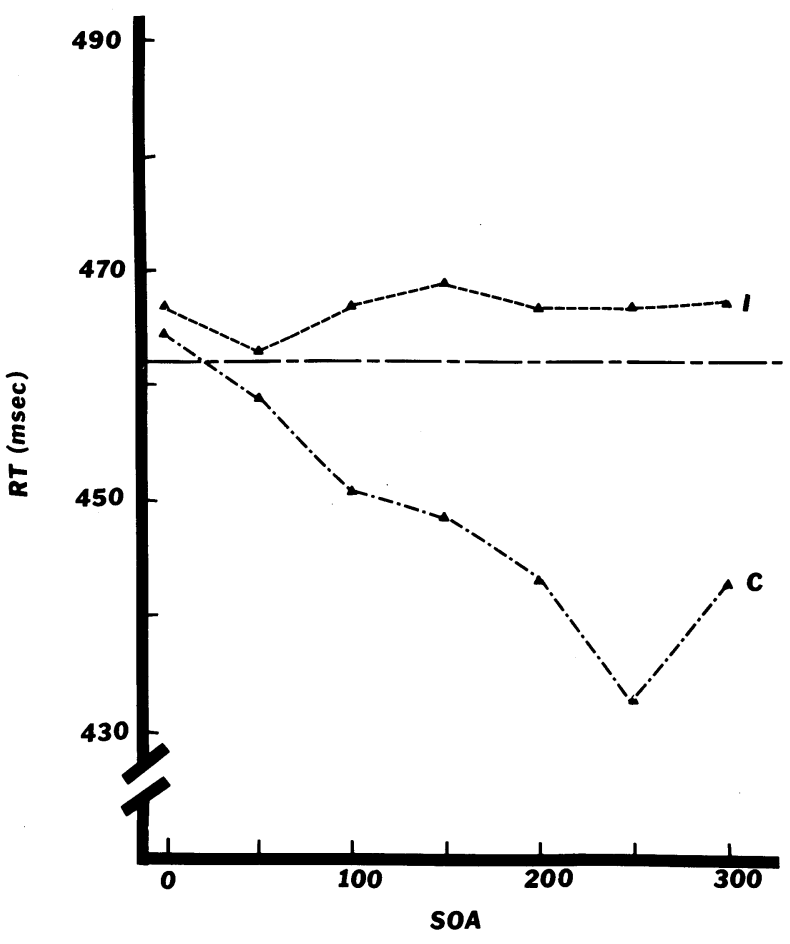

Figure 2. Mean RTs for the "wide" stimulus displays, plotted as a function of SOA.
Table 1

Error Rates

\begin{tabular}{ccccccccc}
\hline & & \multicolumn{7}{c}{ SOA in Milliseconds } \\
\cline { 2 - 8 } $\begin{array}{c}\text { Dis- } \\
\text { play }\end{array}$ & 0 & 50 & 100 & 150 & 200 & 250 & 300 \\
\hline \multirow{2}{*}{$\mathrm{N}$} & $\mathrm{C}$ & .000 & .000 & .003 & .003 & .000 & .000 & .000 \\
& $\mathrm{I}$ & .010 & .014 & .014 & .017 & .017 & .020 & .010 \\
& $\mathrm{C}$ & .000 & .007 & .000 & .000 & .000 & .000 & .000 \\
$\mathrm{~W}$ & $\mathrm{I}$ & .014 & .017 & .024 & .017 & .020 & .027 & .010 \\
\hline
\end{tabular}

Note-Each cell entry is total proportion of errors observed across 42 trials and seven subjects. $N=$ narrow, $W=$ wide; $C=$ congruent, $I=$ incongruent.

evident from both Figures 1 and 2 that there was a greater noise-compatibility effect when the noise digits preceded the target than when they occurred simultaneously with it. Furthermore, the SOA interval producing the maximum differencès in RT between the incongruent and congruent displays was greater for the wide target-noise separations. Both these findings offer support for the continuous flow conception.

\section{Errors}

As is typical in tasks of this type, error rates were quite low. However, as Table 1 indicates, errors (which included premature vocalizations) occurred almost exclusively when incongruent target-noise combinations were displayed. Because of the low frequency of these errors, they were not subjected to any formal analysis.

\section{DISCUSSION}

The tradeoff between spatial separation and response priming effects, illustrated by the three-way interaction of SOA, noisetarget compatibility, and spatial separation and visually apparent in the different shapes of the SOA functions shown in Figures 1 and 2 , is highly consistent with the continuous flow conception of visual information processing described by Eriksen and Schultz (1979). Increasing target-noise separation increases the processing time advantage of the target relative to the noise such that there is less temporal overlap in response priming between the target and noise, yet this overlap can be reinstated by providing the noise elements with a sufficient lead in onset time. It is interesting to note from comparison of Figures 1 and 2 that the additional lead time necessary for compensating for moving the noise elements from 1.1 to $3.3 \mathrm{deg}$ into the periphery seems to be on the order of $100 \mathrm{msec}-\mathrm{a}$ value close to increases in RT obtained in previous experiments (e.g., Eriksen \& Schultz, 1977) in which responses were made to peripheral vs. more centrally located targets. Increased selectivity of visual attention resulting from increasing target-noise separation can thus largely be attributed to processing time advantages for the target as opposed to inhibiting or suppressing the processing of information in the surrounding visual field.

An additional prediction of the continuous flow conception that has thus far received only weak support (Eriksen \& Schultz, 1979 ) is the existence of facilitative priming effects with compatible target-noise combinations as well as response-competition effects when the target and noise specify different responses. The present data do suggest such facilitation, since the RTs for the congruent target-noise combinations are substantially lower than for the distractorless control condition. Unfortunately, the 
data also suggest, by the main effect of SOA, that the mere onset of the noise may have contributed to a general facilitative warning signal effect that would be absent in a distractorless control. While this warning signal effect appears to be relatively small compared with the overall differences among the RTs for the incongruent, control, and congruent displays at a $100-\mathrm{msec}$ SOA (for the narrow display), stronger evidence for facilitation might have been obtained if a "neutral" noise baseline had been included. Additionally, the division of $50 \%$ congruent and $50 \%$ incongruent trials, together with seven response alternatives, leads to a reduction in response uncertainty when the noise appears. While evidence from lexical decision experiments (e.g., Neely, 1977) suggests that expectancies based on conditional probabilities do not become an important influence on decision time unless considerably longer SOA values than those of the present experiment are used (except, perhaps, the $300-\mathrm{msec}$ value), arguments for the existence of purely "automatic" facilitative response priming would be strengthened by use of a task in which the distractors provided no reduction of response uncertainty. These issues are currently under investigation.

\section{REFERENCES}

Bjork, E. L., \& Murray, S. T. On the nature of input channels in visual processing. Psychological Review, 1977, 84, 472-484.

Eriksen, B. A., \& Eriksen, C. W. Effects of noise letters upon the identification of a target letter in a nonsearch task. Perception \& Psychophysics, 1974, 16, 143-149.

Eriksen, C. W., \& Hoffman, J. E. The extent of processing of noise elements during selective encoding from visual displays. Perception \& Psychophysics, 1973, 14, 155-160.
Eriksen, C. W., \& Schultz, D. W. Retinal locus and acuity in visual information processing. Bulletin of the Psychonomic Society, 1977, 9,81-84.

Eriksen, C. W., \& Schultz, D. W. Information processing in visual search: A continuous flow conception and experimental results. Perception \& Psychophysics, 1979, 25, 249-263.

Estes, W. K. Redundancy of noise elements and signals in visual detection of letters. Perception \& Psychophysics, 1974, 16, 53-60.

Flowers, J. H., Warner, J. L., \& Polansky, M. L. Response and encoding factors in ignoring irrelevant information. Memory \& Cognition, 1979, 11, 115-118.

NeELY, J. H. Semantic priming and retrieval from lexical memory: Roles of inhibitionless spreading activation and limited capacity attention. Journal of Experimental Psychology: General, 1977, 106, 116-154.

Santee, J. L. \& Egeth, H. E. Interference in letter identification: A test of feature specific inhibition. Perception \& Psychophysics, 1980, 27, 321-330.

\section{NOTES}

1. The term "sensitivity" is meant to refer both to visual acuity in peripheral vs. control locations and to spatial expectancies.

2. This display methodology involving Os that change into noise elements or targets was employed to reduce apparent' movement.

(Received for publication October 30, 1980.) 